$1-1-1987$

\title{
Beef and sheep farming in the Allegheny highlands : an analysis of alternative management strategies on small farms and farmer reactions
}

Kimberly Reda-Wilson

Follow this and additional works at: https://researchrepository.wvu.edu/ wv_agricultural_and_forestry_experiment_station_bulletins

\section{Digital Commons Citation}

Reda-Wilson, Kimberly, "Beef and sheep farming in the Allegheny highlands : an analysis of alternative management strategies on small farms and farmer reactions" (1987). West Virginia Agricultural and Forestry Experiment Station Bulletins. 694.

https://researchrepository.wvu.edu/wv_agricultural_and_forestry_experiment_station_bulletins/588 @ WVU. It has been accepted for inclusion in West Virginia Agricultural and Forestry Experiment Station Bulletins by an authorized administrator of The Research Repository @ WVU. For more information, please contact ian.harmon@mail.wvu.edu. 


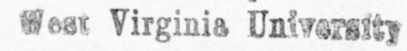

\section{BEEF AND SHEEP FARMING IN THE ALLEGHENY HIGHLANDS:}

An Analysis of Alternative Management Strategies on Small Farms and Farmer Reactions

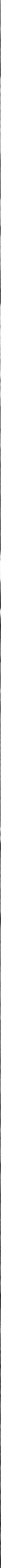




\section{The Authors}

Kimberly Reda-Wilson is former Research Assistant and Robert O. Burton, Jr. is former Assistant Professor, Division of Resource Management; Barton S. Baker is Professor, Division of Plant and Soil Sciences; and Paul E. Lewis is Professor, Division of Animal and Veterinary Sciences, West Virginia University Agricultural and Forestry Experiment Station. RedaWilson is currently Extension Associate, Virginia Polytechnic Institute and State University. Burton is currently Assistant Professor at Department of Agricultural Economics, Kansas State University. Appreciation is expressed to Anwarul Hoque, Dennis K. Smith, and Mary E. Templeton for reviewing an earlier draft. The authors are responsible for any remaining errors.

West Virginia University

Agricultural and Forestry Experiment Station

College of Agriculture and Forestry

Robert H. Maxwell, Director

Morgantown 


\section{Contents}

$\begin{array}{ll}\text { Preface } & \text { iii }\end{array}$

Introduction 1

Research Objectives 1

Selection of Farm Categories 2

Data Sources 2

Farm Descriptions Based on Actual Data 3

Typical Budgets 5

Linear Programming Structure of Typical Farms 6

Comparison of Typical Models to Actual Data 6

Beef/Sheep Models 9

Beef Models and Sheep Models 9

$\begin{array}{ll}\text { Alternative Management Strategies } & 14\end{array}$

Beef Artificial Insemination $\quad 14$

Calf Wintering $\quad 15$

Intensive Sheep 16

$\begin{array}{ll}\text { Labor Hiring } & 17\end{array}$

$\begin{array}{lc}\text { Survey of AHP Farmers } & 18\end{array}$

$\begin{array}{ll}\text { Marketing Beef Cattle } & 18\end{array}$

$\begin{array}{ll}\text { Hiring Labor } & 18\end{array}$

$\begin{array}{ll}\text { Conclusions } & 21\end{array}$

$\begin{array}{ll}\text { References } & 21\end{array}$

$\begin{array}{ll}\text { Appendix } & 23\end{array}$ 


\section{Tables}

1. Selected characteristics of AHP farms, 1978 and 1979

2. Typical full-time AHP beef/sheep farm model 7

3. Typical part-time AHP beef/sheep farm model 8

4. Summary of actual average data for full-time AHP farms and LP model results

5. Summary of actual average data for part-time AHP farms and LP model results

6. Marketing strategies of AHP farmers, $1982 \quad 20$

A-1. AHP variable descriptions and mean values 23

A-2. Machinery inventory for twenty-six full-time AHP farms 24

A-3. Machinery inventory for thirty part-time AHP farms 26

\section{Figures}

1. Marketing strategies of AHP farmers, 1982

2. Number of AHP farms hiring labor and average hours of labor hired per month, 1982

3. Number of AHP farms hiring labor and total hours of labor hired per month, 1982 


\section{Preface}

This bulletin is a summary of research performed under U. S. Department of Agriculture cooperative agreement number 58-32U4-0-203, titled "Resource Characterization of Small Farms and Evaluation of Whole Farm Systems Using Existing Small Farm Data." Additional details of the research may be found in Reda, Kimberly Jane, "An Economic Analysis of Management Strategies on Small, Beef/Sheep Farms in West Virginia," M. S. thesis, West Virginia University, 1984. 


\section{[Blank Page in Original Bulletin]}




\section{Beef and Sheep Farming in the Allegheny Highlands: An Analysis of} Alternative Management Strategies on Small Farms and Farmer Reactions

Kimberly Reda-Wilson, Robert O. Burton, Jr., Barton S. Baker, and Paul E. Lewis

\section{Introduction}

During the past forty years, the agricultural sector of the United States has become highly mechanized, technologically advanced, and eminently productive. This transformation has enabled some farm operators to expand and prosper while providing an abundant food supply (Orden and Smith).

Many farms in West Virginia are located on rough terrain unsuited to highly mechanized, intensive cultivation typical of the larger operations which began to dominate the industry in the 1950s. This structural change in farming is evidenced by the 79 percent decrease in West Virginia farm numbers from 1945-1978 (U.S. Department of Commerce). The remaining small, livestock/ forage units, characteristic of the state's farms, are faced with persistent low income. ${ }^{1}$ Therefore, research is needed to evaluate the income potential for alternative management strategies on small, livestock/forage farms.

\section{Research Objectives}

This research was intended to determine if management practices used by farmers who had participated in the Allegheny Highlands Project (AHP) had led to optimal profit. ${ }^{2}$ The general objective was to evaluate the profitability of a greater use of technology.

The specific objectives of the study were:

1. to establish a profile of characteristics for an average full-time and parttime Allegheny Highlands Project farm;

\footnotetext{
${ }^{1}$ The average net farm income was lower for 60 percent of USDA small farms in West Virginia than the reported non-metropolitan median family income $(\$ 14,800)$ for West Virginia in 1979 (Crecink).

${ }^{2}$ Allegheny Highlands Project, hereafter referred to as AHP or Project, was a ten-year (1970-79) research and demonstrational program designed to revitalize the beef and sheep industries in West Virginia (Baker et al.). The operation, co-sponsored by the West Virginia University (WVU) College of Agriculture and Forestry and the Rockefeller Foundation, was centered in a nine-county area in north-central West Virginia.
} 
2. to develop annual pasture, mixed hay, beef, and sheep enterprise budgets for full-time and part-time farms;

3. to integrate the profile of characteristics and budgets into synthetic wholefarm computer models representing an optimally managed full-time and part-time farm;

4. to prepare budgets and whole-farm models of alternative production techniques;

5. to evaluate the economic feasibility of alternative production techniques;

6. to identify the most promising alternative management systems; and

7. to evaluate the potential for adoption of the more promising alternative management systems from the AHP farm operator's viewpoint.

\section{Selection of Farm Categories}

Based on the statistical technique, discriminant analysis, and the desire to limit the analysis to two groups, $56 \mathrm{AHP}$ farms were classified as either full or part time. ${ }^{3}$ The full-time category was composed of 26 farms whose labor inputs, as originally judged in the Project, were full-time with no outside income or full-time with outside income. The part-time group was composed of 30 farms whose time inputs were part-time with farming as a major activity, part-time with farming as a minor activity, or retired.

\section{Data Sources}

Production, income and expense, and inventory data obtained from AHP were used as the primary data source. Data collection had been coordinated through a computerized record keeping system, the Electronic Farm Accounting Program (ELFAC), ${ }_{4}^{4}$ used by cooperating farmers (Baker et al., pp. 1-2). Data not available from the ELFAC records were obtained from: (1) published research data; (2) estimates of qualified, experienced agricultural scientsts; ${ }^{5}$ (3) research results; and (4) surveys of farmers.

Selected population characteristics for the years 1978 and 1979 are presented in Table A-1. ${ }^{6}$ Since the study was based on a 1979 price level, indexes were used to convert 1978 prices to the 1979 price level prior to averaging (USDA 1981, p. 452). Additional explanations of data sources may be found in the M.S. thesis by Reda.

\footnotetext{
${ }^{3}$ Discriminant analysis was used to evaluate alternative combinations of groups of farms categorized earlier by AHP personnel (Baker et al., pp. 9-10).

${ }^{4}$ Electronic Farm Accounting Program is a Vermont-based operation offered through the Division of Resource Management at WVU.

${ }^{5}$ The authors acknowledge the assistance of Keith Inskeep, animal scientist, West Virginia College of Agriculture and Forestry and Phil Osborne, livestock specialist from the Center for Extension and Continuing Education at WVU.
}

${ }^{6}$ The authors acknowledge the assistance of Randy Martin, former research assistant at WVU. 


\section{Farm Descriptions Based on Actual Data}

The average full-time farm consisted of 480 acres valued at $\$ 60,340$. Based on inventory data, approximately 43.8 percent of the land was used to produce pasture, 13.5 percent for crops (mostly hay), and 42.7 percent for woodland and other uses. Thus, for purposes of livestock production, land was predominantly used for pasture. A total of $\$ 2,500$ per farm was spent for lime $(\$ 486.00)$ and fertilizer $(\$ 2,014)$ or $\$ 9.09$ per acre of pasture and tillable land. The average full-time farmer owned 1 baler, 4 pieces of other hay harvesting equipment, 1 piece of corn harvesting equipment, 3 tillage implements, 3 tractors, and 2 wagons (Table A-2).

The majority of full-time producers raised a combination of beef and sheep (Table 1). The total number of animal units per farm averaged 109, of which approximately 76 percent was beef, 21 percent sheep, and 3 percent other livestock. The full-time farmer spent approximately $\$ 6.00$ for veterinary expenses, $\$ 3.00$ for salt and minerals, and $\$ 1.00$ for livestock supplies per animal unit. Approximately $\$ 7.00$ [ $\$ 11.00$ ] was spent per cow [ewe] for beef [sheep] concentrates and $\$ 636$ worth of hay per farm was purchased.

Table 1

Selected characteristics of AHP farms, 1978 and 1979.a

\begin{tabular}{llrr}
\hline \hline & & \multicolumn{2}{c}{ Quantity } \\
\cline { 3 - 4 } Category & Unit & Full time & Part time \\
\hline Farms: & Number & 26 & 30 \\
Beef/sheep farms & Number & 18 & 7 \\
Beef farms & Number & 7 & 21 \\
Sheep farms & Number & 1 & 2 \\
Land Inventory & & & \\
Crop & Acres & 65 & 45 \\
Pasture & Acres & 210 & 94 \\
Woodland & Acres & 201 & 103 \\
Other & Acres & 4 & 3 \\
Pasture yields per acre & TDM & 1.95 & 1.75 \\
Hay yields per acrec & TDM & 2.88 & 2.76 \\
Beef statistics: & & & \\
Cow & Number & 66 & 32 \\
Cow death rate & Percent & 2 & 2 \\
Calf birtindate from Nov. 1 & Days & 130 & 124 \\
205-day weight & Pounds & 465 & 466 \\
Marketable calf crop & Percent & 88 & 86 \\
Calf sale date from Nov. 1 & Days & 342 & 344 \\
Calf weight & Pounds & 483 & 472 \\
Value per calf sold & Dollars & 349 & 334 \\
& & & (continued) \\
& S & &
\end{tabular}


Table 1 (continued).

\begin{tabular}{llrr}
\hline \hline & & \multicolumn{2}{c}{ Quantity } \\
\cline { 3 - 4 } Category & Unit & Full time & Part time \\
\hline Sheep Statistics: & & & \\
Ewe & Number & 137 & 56 \\
Sheep death rate & Percent & 8 & 11 \\
Lamb birthdate from Nov. 1 & Days & 72 & 54 \\
Marketable lamb crop & Percent & 120 & 121 \\
Lamb sale date from Jan. 1 & Days & 279 & 240 \\
Lamb weight & Pounds & 97 & 90 \\
Value per lamb sold & Dollars & 59 & 53 \\
Livestock expenses: & & & \\
Veterinarian and medical & Dollars & 655 & 177 \\
Salt and minerals & Dollars & 305 & 104 \\
Beef concentrate & Dollars & 430 & 295 \\
Sheep concentrate & Dollars & 1,553 & 461 \\
Livestock supplies & Dollars & 128 & 50 \\
Farm finances: & & & \\
Operating receipts & Dollars & 32,871 & 9,678 \\
Operating expenses & Dollars & 24,765 & 7,859 \\
Assets & Dollars & 128,806 & 74,893 \\
Liabilities & Dollars & 12,970 & 10,174 \\
\hline
\end{tabular}

aWhen calculating means missing data were ignored. Consequently, average cow [ewe] numbers are based only on farms which produce cattle [sheep].

bTons of dry matter.

cThese hay yields include .35 and .33 tons of dry matter which were grazed on the full-time and part-time farms, respectively.

Approximately 67 percent of the operating receipts came from the sale of beef, 29 percent from sheep, 2.5 percent from wool, and 1.5 percent from hay and grain. Operating receipts minus operating expenses were $\$ 8,106$ per farm or $\$ 74$ per animal unit.

Other data used to characterize an average full-time AHP farm included: 32 pieces of equipment per farm; $\$ 14,632$, depreciated value of machinery; $\$ 568$, repairs to machinery; $\$ 2,740$, fuel and operation of trucks and tractors; $\$ 305$, hired machinery; and $\$ 870$, repairs to real estate. The net worth of a full-time AHP farm was $\$ 115,836$.

The average part-time farm consisted of 245 acres. Approximately 38.4 percent of the land was used to produce pasture, 18.4 percent for crops (mostly hay), and 43.2 percent for woodland and other uses. Thus, land use patterns were similar for both groups of farms. A total of $\$ 857$ was spent for lime $(\$ 170)$ and fertilizer $(\$ 687)$ or $\$ 6.17$ per acre of pasture and tillable land. The average part-time farmer owned 1 baler, 3 pieces of other hay harvesting equipment, 1 piece of corn harvesting equipment, 2 tillage implements, 2 tractors, and 1 wagon (Table A-3). 
Unlike the full-time farmers, the majority of part-time farmers raised only beef. The total number of animal units per farm averaged 44, of which approximately 77 percent was beef, 19 percent sheep, and 4 percent other livestock. The part-time farmer spent approximately $\$ 4.00$ for veterinary expenses, $\$ 2.00$ for salt and minerals, and $\$ 1.00$ for livestock supplies per animal unit. Aproximately $\$ 9.00$ [8.00] was spent per cow [ewe] for beef [sheep] concentrates and $\$ 172$ worth of hay was purchased.

Approximately 78 percent of the total operating receipts came from the sale of beef, 17 percent from sheep, 2 percent from wool, and 3 percent from hay and grain. Operating receipts minus operating expenses were $\$ 1,819$ per farm or $\$ 41$ per animal unit.

Other data from part-time farms included: 19.5 pieces of equipment per farm; $\$ 8,954$, depreciated value of machinery; $\$ 247$, repairs to machinery; $\$ 1,567$, fuel and operation of trucks and tractors; $\$ 25$, hired machinery; and $\$ 440$, repairs to real estate. The average net worth of a part-time AHP farm was $\$ 64,719$.

\section{Typical Budgets}

Annual production levels, costs, and returns to fixed resources and feed ${ }^{7}$ were estimated for pasture, mixed hay, beef, and sheep. The budgets representing a full-time farm reflected the following: (1) An average pasture yield of 1.95 tons of dry matter per acre for an annual outlay (variable costs) of $\$ 14.19$; (2) An average hay yield of 2.88 tons of dry matter per acre for an annual outlay of $\$ 58.19$; (3) A 60 -cow herd producing 53 calves per year whose average market weight was 483 pounds. The annual returns to fixed resources and feed per cow were $\$ 244.35$; and (4) A 150 -ewe flock producing 180 lambs whose average market weight was 97 pounds. The annual returns to fixed resources and feed per ewe were $\$ 53.67$.

Statistics for the part-time farm were: (1) Pasture yield of 1.75 tons of dry matter at $\$ 9.19$ per acre; (2) Hay yield of 2.76 tons of dry matter at $\$ 56.45$ per acre; (3) A 30-cow herd, 26 calves, 472 pounds per marketed calf, and $\$ 211.81$ returns to fixed resources and feed per cow; and (4) A 50-ewe flock, 61 lambs, 90 pounds per marketed lamb, and $\$ 43.17$ returns to fixed resources and feed per ewe.

${ }^{7}$ Returns to fixed resources and feed (total revenue minus variable costs) and returns to land, risk, management, and feed (total revenue minus variable and fixed costs) were both assessed in the budgets. Feed costs were accounted for in the whole-farm computer models so feed costs were not subtracted from the budgeted returns reported in this selection. Since many of the producers represented by the models are known to be reluctant to borrow money for farm operations, interest on operating capital was not included as a cash (variable) cost. 


\section{Linear Programming Structure of Typical Farms}

Given the AHP farmers available land, labor, operating capital and facilities, and restricting enterprises to those commonly found on farms in 1979, a linear programming (LP) model $^{8}$ was used to estimate potential returns for each farm category, assuming an optimally managed production process. The enterprise input-output relationships were incorporated into a matrix table. The objective in solving the model was to determine the cropping and livestock enterprise combination which would maximize returns to fixed resources. Fixed resources included owned land, on-farm labor, capital, machinery, buildings, equipment, and management.

Crop and livestock production coefficients, costs, and returns for a full-time farm were derived from enterprise budgets. The activities representative of the four most common enterprises included in the full-time, typical beef/sheep farm model were pasture, hay, beef, and sheep (Table 2). Other activities included in this base model were those allowing for the purchase of inputs (corn and hay) and disposal of intermediate products (pasture and hay). Transfer columns were designed to depict the availability of pasture from one month to another. Land, capital, and labor restrictions during the critical seasons were included.$^{9}$ The production activities were arranged as a series of columns. The resources required for these production alternatives were listed by row (Tables 2 and 3).

Depending on the personal objectives of the manager, restraints may be subjective in nature. Thus, livestock models representing the production of only beef or only sheep were analyzed. The full-time, typical sheep [beef] model was prepared by deleting the beef (sheep and corn purchase ${ }^{10}$ ) activity from the format of the original beef/sheep model.

Based on part-time AHP data, the LP model was designed to reflect a typical part-time beef/sheep farm's level of performance (Table 3). Part-time models representing only beef or only sheep production were also developed.

\section{Comparison of Typical Models to Actual Data}

In order to assess the practical validity of the LP models, the models were compared to actual data. This assessment is beneficial because the more closely the model results conform to actual statistics the more confidence may be placed in recommendations based on the models.

${ }^{8} \mathrm{Linear}$ programming is a mathematical technique providing for the solution of a linear objective function subject to a set of resource limitations (Beneke and Winterboer).

${ }^{9}$ Land and capital constraints were taken from AHP data. Labor requirements were based on information from Purdue University (Coop. Ext. Serv.) and Willow Bend Demonstrational Farm.

${ }^{10}$ The purchase of corn is not necessary for the production of beef cattle. 
Table 2

Typical full-time AHP beef/sheep farm model. ${ }^{a}$

\begin{tabular}{|c|c|c|c|c|c|c|c|c|c|c|c|c|c|c|c|c|c|}
\hline (Units) & & $\begin{array}{l}\text { Growpast } \\
\text { (Ac) }\end{array}$ & $\begin{array}{l}\text { Growhay } \\
\text { (AC) }\end{array}$ & $\begin{array}{l}\text { Sumhay } \\
\text { (Lb dm) }\end{array}$ & $\begin{array}{l}\text { Buycorn } \\
\text { (Cwt) }\end{array}$ & $\begin{array}{l}\text { Buyhay } \\
\text { (Tn) }\end{array}$ & $\begin{array}{l}\text { Grow corn } \\
\text { for Cow }\end{array}$ & $\begin{array}{l}\text { Grow } \\
\text { silage } \\
\text { for Ewe }\end{array}$ & $\begin{array}{c}\text { Sellpast } \\
\text { (Cow) }\end{array}$ & $\begin{array}{c}\text { Sellhay } \\
(T n)\end{array}$ & $\begin{array}{l}\text { Apr.- } \\
\text { May } \\
\text { (Lb dm) (I }\end{array}$ & $\begin{array}{l}\text { May- } \\
\text { June } \\
(\text { Lb dm)( }\end{array}$ & $\begin{array}{l}\text { June- } \\
\text { July } \\
\text { (Lb dm)( }\end{array}$ & $\begin{array}{l}\text { July- } \\
\text { Aug. } \\
\text { (Lb dm) }\end{array}$ & $\begin{array}{l}\text { Aug.- } \\
\text { Sept. } \\
\text { (Lb dm) }\end{array}$ & $\begin{array}{l}\text { Sept- } \\
\text { Oct. } \\
\text { (Lb dm) }\end{array}$ & $\mathrm{RHS}^{\mathrm{b}}$ \\
\hline$(\$)$ & Objf & -14.19 & -58.19 & & -4.47 & -50.01 & 244.35 & 53.67 & 20.40 & 50.00 & & & & & & & $N$ \\
\hline (Ac) & Hayland & & 1.00 & & & & & & & & & & & & & & 65 \\
\hline$(\mathrm{Ac})$ & Totland & 1.00 & 1.00 & & & & & & & & & & & & & & 275 \\
\hline (Cwt) & Corn & & & & 1.00 & & & 1.00 & & & & & & & & & $\leq$ \\
\hline (Lb dm) & Hay & & $-5,060.00$ & 1.00 & & $-1,760.00$ & $5,165.00$ & 527.00 & & $1,760.00$ & & & & & & & $\leq$ \\
\hline$(\mathrm{Hr})$ & $\begin{array}{l}\text { Ann- } \\
\text { labor }\end{array}$ & .42 & 10.46 & & & & 8.00 & 5.00 & & & & & & & & & $\leq$ \\
\hline$(\mathrm{Hr})$ & Calvimb & & & & & & 2.88 & 3.23 & & & & & & & & & 600 \\
\hline$(\mathrm{Hr})$ & $\begin{array}{l}\text { Jun- } \\
\text { labor }\end{array}$ & & 5.23 & & & & .28 & .10 & & & & & & & & & $\leq \quad 300$ \\
\hline$(\$)$ & Opcap & 14.19 & 58.19 & & 4.47 & 50.01 & 71.33 & 18.02 & & & & & & & & & $\leq 24,765$ \\
\hline (Lb dm) & Aprfeed & -117.00 & & & & & & & & & 1.0 & & & & & & $\leq \quad 0$ \\
\hline (Lb dm) & Mayfeed & $-1,229.00$ & & & & & $1,489.00$ & 235.00 & $1,200.00$ & & -.9 & 1.0 & & & & & $\leq$ \\
\hline$(\mathrm{Lb} d \mathrm{dm})$ & Junfeed & -819.00 & & & & & $1,489.00$ & 235.00 & $1,200.00$ & & & -.9 & 1.0 & & & & $\leq$ \\
\hline (Lb dm) & Julyfeed & -683.00 & & & & & $1,647.00$ & 272.00 & $1,200.00$ & & & & -.9 & $1: 0$ & & & $\leq$ \\
\hline (Lb dm) & ) Augfeed & -546.00 & & & & & $1,647.00$ & 272.00 & $1,200.00$ & & & & & -.9 & 1.0 & & $\leq$ \\
\hline (Lb dm) & $\begin{array}{l}\text { Sept- } \\
\text { feed }\end{array}$ & -312.00 & -700.00 & & & & $1,910.00$ & 333.00 & $1,200.00$ & & & & & & -.9 & 1.0 & $\leq$ \\
\hline (Lb dm) & Octfeed & -195.00 & & -1.00 & & & $1,910.00$ & 333.00 & $1,200.00$ & & & & & & & -.9 & $\leq$ \\
\hline
\end{tabular}

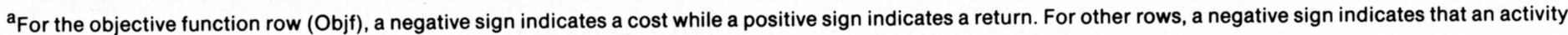
furnishes a resource while a positive sign indicates an activity uses a resource.

${ }^{b}$ Maximum Resources Available. 
Table 3

Typical part-time AHP beef/sheep farm model. ${ }^{\mathrm{a}}$

\begin{tabular}{|c|c|c|c|c|c|c|c|c|c|c|c|c|c|c|c|c|c|c|}
\hline (Units) & & $\begin{array}{l}\text { Growpast } \\
\text { (Ac) }\end{array}$ & $\begin{array}{l}\text { Growhay } \\
\text { (Ac) }\end{array}$ & $\begin{array}{c}\text { Sumhay } \\
(\text { Lb dm) }\end{array}$ & $\begin{array}{l}\text { Buycorn } \\
\text { (Cwt) }\end{array}$ & $\begin{array}{c}\text { Buyhay } \\
\text { (Tn) }\end{array}$ & $\begin{array}{c}\text { Grow corn } \\
\text { for Cow }\end{array}$ & $\begin{array}{c}\text { Grow } \\
\text { silage } \\
\text { for Ewe }\end{array}$ & $\begin{array}{l}\text { Sellpast } \\
\text { (Cow) }\end{array}$ & $\begin{array}{c}\text { Sellhay } \\
\text { (Tn) }\end{array}$ & $\begin{array}{c}\text { Apr.- } \\
\text { May } \\
\text { (Lb dm) }\end{array}$ & $\begin{array}{l}\text { May- } \\
\text { June } \\
\text { (Lb dm) ( }\end{array}$ & $\begin{array}{l}\text { June- } \\
\text { July } \\
\text { (Lb dm) }\end{array}$ & $\begin{array}{l}\text { July- } \\
\text { Aug. } \\
\text { (Lb dm) }\end{array}$ & $\begin{array}{l}\text { Aug.- } \\
\text { Sept. } \\
\text { (Lb dm) }\end{array}$ & $\begin{array}{l}\text { Sept- } \\
\text { Oct. } \\
\text { (Lb dm) }\end{array}$ & & $\mathrm{RHS}^{\mathrm{b}}$ \\
\hline (\$) & Objf & -9.19 & -56.45 & & -4.47 & -50.01 & 211.81 & 43.17 & 20.40 & 50.00 & & & & & & & $N$ & 0 \\
\hline (Ac) & Hayland & & 1.00 & & & & & & & & & & & & & & $\leq$ & 45 \\
\hline (Ac) & Totland & 1.00 & 1.00 & & & & & & & & & & & & & & $\leq$ & 139 \\
\hline (Cwt) & Corn & & & & 1.00 & & & 1.00 & & & & & & & & & $\leq$ & 0 \\
\hline (Lb dm) & Hay & & $-4,860.00$ & 1.00 & & $-1,760.00$ & $5,165.00$ & 530.00 & & $-1,760.00$ & & & & & & & $\leq$ & 0 \\
\hline$(\mathrm{Hr})$ & $\begin{array}{l}\text { Ann- } \\
\text { labor }\end{array}$ & .42 & 10.46 & & & & 8.00 & 5.00 & & & & & & & & & $\leq$ & 0 \\
\hline$(\mathrm{Hr})$ & Calvimb & & & & & & 2.88 & 3.23 & & & & & & & & & $\leq$ & 360 \\
\hline (Hr) & $\begin{array}{l}\text { Jun- } \\
\text { labor }\end{array}$ & & 5.23 & & & & .28 & .10 & & & & & & & & & $\leq$ & 180 \\
\hline (\$) & Opcap & 9.19 & 56.45 & & 4.47 & 50.01 & 85.74 & 22.09 & & & & & & & & & $\leq$ & 7,859 \\
\hline (Lb dm) & Aprfeed & -105.00 & & & & & & & & & 1.0 & & & & & & $\leq$ & 0 \\
\hline (Lb dm) & Mayfeed & $-1,103.00$ & & & & & $1,486.00$ & 237.00 & $1,200.00$ & & -.9 & 1.0 & & & & & $\leq$ & 0 \\
\hline (Lb dm) & Junfeed & -735.00 & & & & & $1,486.00$ & 237.00 & $1,200.00$ & & & -.9 & 1.0 & & & & $\leq$ & 0 \\
\hline (Lb dm) & Julyfeed & -613.00 & & & & & $1,641.00$ & 274.00 & $1,200.00$ & & & & -.9 & 1.0 & & & $\leq$ & 0 \\
\hline (Lb dm) & Augfeed & -490.00 & & & & & $1,641.00$ & 274.00 & $1,200.00$ & & & & & -.9 & 1.0 & & $\leq$ & 0 \\
\hline (Lb dm) & $\begin{array}{l}\text { Sept - } \\
\text { feed }\end{array}$ & -280.00 & -661.00 & & & & $1,898.00$ & 336.00 & $1,200.00$ & & & & & & -.9 & 1.0 & $\leq$ & 0 \\
\hline (Lb dm) & Octfeed & -175.00 & & -1.00 & & & $1,898.00$ & 336.00 & $1,200.00$ & & & & & & & -.9 & $\leq$ & 0 \\
\hline
\end{tabular}

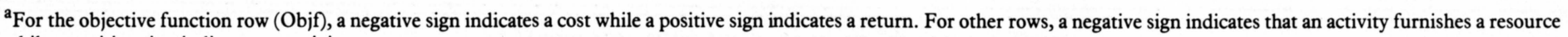
while a positive sign indicates an activity uses a resource.

${ }^{\mathrm{b}}$ Maximum Resources Available. 


\section{Beef/Sheep Models}

The acres of pasture and hay in the full-time beef/sheep model F-1 solution were 101 and 83 percent, respectively, of actual production levels for each enterprise (Table 4). The number of cows and ewes were 97 and 96 percent, respectively, of actual production quantities. The modeled value of hay purchased was approximately four times greater than the actual value, while net income was three percent greater than the corresponding figure in actual data.

Differences between actual and modeled results for the part-time beef/sheep model P-1 were more extreme than those for the full-time group (Table 5). Measured as a percentage of actual 1979 production, differences included: acres of pasture, 137 percent; acres of hay, 80 percent; number of cows, 59 percent; number of sheep, 188 percent; and net income, 140 percent. Net income figures were pointedly dissimilar as a result of the income and costs associated with a larger number of sheep in the optimal solution than actual data and modeled income from hay sales which was approximately four times greater than actual income from hay sales.

\section{Beef Models and Sheep Models}

Differences between actual and modeled data for the full-time, all beef model F-2, measured as a percentage of actual data, included: acres of pasture, 108 percent; acres of hay, 70 percent; and number of cows, 96 percent. The greatest disparity between actual and model data was with expenses for purchased hay and net income, which were more than fifteen and nine times greater, respectively, than actual data. Since the modeled production was so different from actual production, there is reason to suspect the income and cost variables used to model the full-time beef farm and/or the validity of the actual data.

Differences between the optimal solutions of the typical beef model P-2 and actual production levels of an average part-time, al1 beef farm were not as great as those for the full-time category. Measured as a percentage of actual data, they included acres of pasture, 108 percent; acres of hay, 67 percent; number of cows, 106 percent; value of hay purchased, 375 percent; and net income, 227 percent.

Due to the low number of sheep farms, a comparison was not made between model F-3 and P-3 results and actual data.

When interpreting the models' results, one should keep in mind the following considerations: the AHP cooperator's primary farming objective was not always profit; the average price received per CWT of calf was higher in 1979 than in the eight preceding or two following years; ${ }^{11}$ and operating capital was a restraining resource only in LP models P-10 and P-12.

${ }^{11}$ Calf prices per CWT in years $1971-81$ were as follows: $\$ 36.80, \$ 46.30, \$ 57.50, \$ 35.70, \$ 27.40$, $\$ 31.70, \$ 34.50, \$ 57.90, \$ 79.50, \$ 79.30$, and $\$ 66.90$ (USDA and WVDA). 
Table 4

Summary of actual average data for full-time AHP farms and LP model results. ${ }^{\mathrm{a}}$

\begin{tabular}{|c|c|c|c|c|c|c|c|c|c|c|c|}
\hline $\begin{array}{c}\text { Category } \\
\text { (unit) }\end{array}$ & $\begin{array}{c}\text { Net } \\
\text { income } \\
\text { (dollars) }\end{array}$ & $\begin{array}{l}\text { Cow } \\
\text { (head) }\end{array}$ & $\begin{array}{c}\text { Ewe } \\
\text { (head) }\end{array}$ & $\begin{array}{c}\text { Pasture } \\
\text { (acres) }\end{array}$ & $\begin{array}{c}\text { Hay } \\
\text { (acres) }\end{array}$ & $\begin{array}{l}\text { Corn } \\
\text { purch. } \\
\text { (cwt) }\end{array}$ & $\begin{array}{c}\text { Hay } \\
\text { purch. } \\
\text { (dollars) }\end{array}$ & $\begin{array}{c}\text { Hay } \\
\text { sold } \\
\text { (dollars) }\end{array}$ & $\begin{array}{l}\text { Pasture } \\
\text { sold } \\
\text { (dollars) }\end{array}$ & $\begin{array}{l}\text { Labor } \\
\text { hired for } \\
\text { calving/ } \\
\text { lambing } \\
\text { (dollars) }\end{array}$ & $\begin{array}{l}\text { Labor } \\
\text { hired } \\
\text { for hay } \\
\text { harvesting } \\
\text { (dollars) }\end{array}$ \\
\hline \multicolumn{12}{|l|}{ Project data: } \\
\hline All farms & 8,106 & 66 & 137 & 210 & 65 & UN & 636 & 218 & UN & UN & UN \\
\hline Beef/sheep farms & 11,205 & 60 & 139 & 221 & 63 & UN & 813 & 110 & UN & UN & UN \\
\hline Beef farms & 1,057 & 83 & NA & 206 & 76 & UN & 270 & 515 & UN & UN & UN \\
\hline \multicolumn{12}{|l|}{ Model results: } \\
\hline$(F-1)$ & 11,497 & 58 & 134 & 223 & 52 & 134 & 3,073 & 0 & 0 & NA & NA \\
\hline$(F-2)$ & 9,203 & 80 & NA & 222 & 53 & NA & 4,112 & 0 & 0 & NA & NA \\
\hline$(F-3)$ & 9,845 & NA & 186 & 79 & 54 & 186 & 0 & 4,954 & 0 & NA & NA \\
\hline$(F-4)$ & 11,320 & 58 & 134 & 223 & 52 & 134 & 3,073 & 0 & 0 & NA & NA \\
\hline$(F-5)$ & 8,957 & 80 & NA & 222 & 53 & NA & 4,112 & 0 & 0 & NA & NA \\
\hline$(F-6)$ & 12,663 & 47 & 139 & 223 & 52 & 139 & 2,673 & 0 & 0 & NA & NA \\
\hline$(F-7)$ & 10,176 & 66 & NA & 221 & 54 & NA & 3,606 & 0 & 0 & NA & NA \\
\hline$(F-8)$ & 7,507 & NA & 186 & 78 & 56 & 186 & 0 & 4,954 & 0 & NA & NA \\
\hline (F-9) & 5,352 & NA & 186 & $65^{b}$ & NA & 186 & 3,115 & NA & 245 & NA & NA \\
\hline$(F-10)$ & 15,786 & 0 & 459 & 210 & 65 & 459 & 0 & 2,477 & 0 & 2,276 & 222 \\
\hline$(F-11)$ & 9,928 & 77 & NA & 210 & 65 & NA & 1,938 & 0 & 0 & 0 & 160 \\
\hline$(F-12)$ & 15,786 & NA & 459 & 210 & 65 & 459 & 0 & 2,477 & 0 & 2,276 & 222 \\
\hline
\end{tabular}


Table 4. Continued

\begin{tabular}{|c|c|c|c|c|c|c|c|c|c|c|}
\hline \multirow[b]{2}{*}{$\begin{array}{l}\text { Resource } \\
\text { (unit) }\end{array}$} & \multicolumn{2}{|c|}{ Hayland } & \multicolumn{2}{|c|}{ Total land } & \multirow{2}{*}{$\begin{array}{l}\text { Annual } \\
\text { laborc } \\
\text { (hours) }\end{array}$} & \multicolumn{2}{|c|}{$\begin{array}{l}\text { Calving and } \\
\text { lambing labor }\end{array}$} & \multicolumn{2}{|c|}{$\begin{array}{l}\text { Hay harvesting } \\
\text { labor }\end{array}$} & \multirow{2}{*}{$\begin{array}{l}\text { Operating } \\
\text { capital } \\
\text { (dollars) }\end{array}$} \\
\hline & $\begin{array}{l}\text { Amount } \\
\text { (acres) }\end{array}$ & $\begin{array}{l}\text { Shadow price } \\
\text { (dollars) }\end{array}$ & $\begin{array}{l}\text { Amount } \\
\text { (acres) }\end{array}$ & $\begin{array}{l}\text { Shadow price } \\
\text { (dollars) }\end{array}$ & & $\begin{array}{l}\text { Amount } \\
\text { (hours) }\end{array}$ & $\begin{array}{l}\text { Shadow price } \\
\text { (dollars) }\end{array}$ & $\begin{array}{l}\text { Amount } \\
\text { (hours) }\end{array}$ & $\begin{array}{l}\text { Shadow price } \\
\text { (dollars) }\end{array}$ & \\
\hline \multicolumn{11}{|l|}{ Project data: } \\
\hline All farms & 65 & NA & 275 & NA & UN & UN & NA & UN & NA & 24,765 \\
\hline Beef/sheep farms & 63 & NA & 284 & NA & UN & UN & NA & UN & NA & 27,553 \\
\hline Beef farms & 76 & NA & 282 & NA & UN & UN & NA & UN & NA & 20,173 \\
\hline \multicolumn{11}{|l|}{ Model results: } \\
\hline$(F-1)$ & 52 & NA & 275 & 12 & 1,768 & 600 & 6 & 300 & 15 & 16,398 \\
\hline$(F-2)$ & 53 & NA & 275 & 18 & 1,289 & 230 & NA & 300 & 14 & 16,059 \\
\hline$(F-3)$ & 54 & NA & 133 & NA & 1,525 & 600 & 8 & 300 & 17 & 8,426 \\
\hline$(F-4)$ & 52 & NA & 275 & 10 & 1,653 & 600 & 6 & 300 & 15 & 16,609 \\
\hline$(F-5)$ & 53 & NA & 275 & 17 & 1,129 & 230 & NA & 300 & 15 & 16,351 \\
\hline$(F-6)$ & 52 & NA & 275 & 20 & 1,753 & 600 & 5 & 300 & 14 & 16,962 \\
\hline$(F-7)$ & 54 & NA & 275 & 24 & 1,241 & 211 & NA & 300 & 13 & 16,832 \\
\hline$(F-8)$ & 54 & NA & 133 & NA & 1,525 & 600 & 8 & 300 & 9 & 10,764 \\
\hline (F-9) & $65^{b}$ & 4 & 65 & NA & 956 & 600 & 9 & 19 & NA & 8,215 \\
\hline$(F-10)$ & 65 & 45 & 275 & 38 & 3,061 & 1,482 & 3 & 386 & 3 & 19,574 \\
\hline$(F-11)$ & 65 & 61 & 275 & 19 & 1,383 & 221 & NA & 362 & 3 & 14,343 \\
\hline$(F-12)$ & 65 & 45 & 275 & 38 & 3,061 & 1,482 & 3 & 386 & 3 & 19,574 \\
\hline
\end{tabular}

aUN represents "unavailable." NA represents "not applicable." Actual data are an average of 1978 and 1979 data; 1978 dollar values were updated to the 1979 price level. Models F-1 through F-3 represent typical farm situations: (F-1) beef/sheep; (F-2) beef; and (F-3) sheep. Models F-4 through F-12 represent alternative management options: (F-4) beef artificial insemination program for a beef/sheep farm; $(F-5)$ beef artificial insemination program for a beef farm; $(F-6)$ calf wintering program for a beef/sheep farm; (F-7) calf wintering program for a beef farm; (F-8) typical sheep model with fixed cost for hay production; (F-9) intensive sheep model; (F-10) typical beef/sheep model with labor hiring activities for hay harvest and calving/lambing seasons; (F-11) typical beef model with labor hiring activities for hay harvest and calving seasons; ( $F-12$ ) typical sheep model with labor hiring activities for hay harvest and lambing seasons.

bThis was pasture produced on hayland.

'Since no constraint was specified in the model, no shadow price emerged. The figure represents the total amount of labor used, including hired labor. 
Table 5

Summary of actual average data for part-time AHP farms and LP model results. ${ }^{\mathrm{a}}$

\begin{tabular}{|c|c|c|c|c|c|c|c|c|c|c|c|}
\hline $\begin{array}{c}\text { Category } \\
\text { (unit) }\end{array}$ & $\begin{array}{c}\text { Net } \\
\text { income } \\
\text { (dollars) }\end{array}$ & $\begin{array}{l}\text { Cow } \\
\text { (head) }\end{array}$ & $\begin{array}{c}\text { Ewe } \\
\text { (head) }\end{array}$ & $\begin{array}{c}\text { Pasture } \\
\text { (acres) }\end{array}$ & $\begin{array}{c}\text { Hay } \\
\text { (acres) }\end{array}$ & $\begin{array}{l}\text { Corn } \\
\text { purch. } \\
\text { (cwt) }\end{array}$ & $\begin{array}{c}\text { Hay } \\
\text { purch. } \\
\text { (dollars) }\end{array}$ & $\begin{array}{c}\text { Hay } \\
\text { sold } \\
\text { (dollars) }\end{array}$ & $\begin{array}{l}\text { Pasture } \\
\text { sold } \\
\text { (dollars) }\end{array}$ & $\begin{array}{l}\text { Labor } \\
\text { hired for } \\
\text { calving/ } \\
\text { lambing } \\
\text { (dollars) }\end{array}$ & $\begin{array}{l}\text { Labor } \\
\text { hired } \\
\text { for hay } \\
\text { harvesting } \\
\text { (dollars) }\end{array}$ \\
\hline \multicolumn{12}{|l|}{ Project data: } \\
\hline All farms & 1,819 & 32 & 56 & 94 & 45 & UN & 172 & 219 & UN & UN & UN \\
\hline Beef/sheep farms & 3,641 & 32 & 50 & 78 & 40 & UN & 82 & 24 & UN & UN & UN \\
\hline Beef farms & 1,745 & 33 & NA & 98 & 49 & UN & 181 & 288 & UN & UN & UN \\
\hline \multicolumn{12}{|l|}{ Model results: } \\
\hline$(P-1)$ & 5,082 & 19 & 94 & 107 & 32 & 94 & 0 & 85 & 0 & NA & NA \\
\hline$(P-2)$ & 3,969 & 35 & NA & 106 & 33 & NA & 678 & 0 & 0 & NA & NA \\
\hline$(P-3)$ & 4,778 & NA & 111 & 54 & 32 & 111 & 0 & 2,780 & 0 & NA & NA \\
\hline$(P-4)$ & 4,881 & 19 & 94 & 107 & 32 & 94 & 0 & 85 & 0 & NA & NA \\
\hline$(P-5)$ & 3,605 & 35 & NA & 106 & 33 & NA & 678 & 0 & 0 & NA & NA \\
\hline$(P-6)$ & 5,506 & 16 & 95 & 107 & 32 & 95 & 0 & 214 & 0 & NA & NA \\
\hline$(P-7)$ & 4,720 & 29 & NA & 106 & 33 & NA & 458 & 0 & 0 & NA & NA \\
\hline$(P-8)$ & 3,375 & NA & 111 & 54 & 32 & 111 & 0 & 2,780 & 0 & NA & NA \\
\hline$(P-9)$ & 2,047 & NA & 111 & $45^{b}$ & NA & 111 & 1,879 & NA & 252 & NA & NA \\
\hline$(P-10)$ & 6,100 & 0 & 154 & 74 & 45 & 154 & 0 & 3,890 & 0 & 356 & 183 \\
\hline$(\mathrm{P}-11)$ & 4,746 & 32 & NA & 94 & 45 & NA & 0 & 1,472 & 0 & 0 & 165 \\
\hline$(\mathrm{P}-12)$ & 6,100 & NA & 154 & 74 & 45 & 154 & 0 & 3,890 & 0 & 356 & 183 \\
\hline
\end{tabular}


Table 5. Continued

\begin{tabular}{|c|c|c|c|c|c|c|c|c|c|c|}
\hline \multirow[b]{2}{*}{$\begin{array}{l}\text { Resource } \\
\text { (Unit) }\end{array}$} & \multicolumn{2}{|c|}{ Hayland } & \multicolumn{2}{|c|}{ Total land } & \multirow{2}{*}{$\begin{array}{l}\text { Annual } \\
\text { laborc } \\
\text { (hours) }\end{array}$} & \multicolumn{2}{|c|}{$\begin{array}{l}\text { Calving and } \\
\text { lambing labor }\end{array}$} & \multicolumn{2}{|c|}{$\begin{array}{l}\text { Hay harvesting } \\
\text { labor }\end{array}$} & \multirow{2}{*}{$\begin{array}{l}\text { Operating } \\
\text { capital } \\
\text { (dollars) }\end{array}$} \\
\hline & $\begin{array}{l}\text { Amount } \\
\text { (acres) }\end{array}$ & $\begin{array}{l}\text { Shadow price } \\
\text { (dollars) }\end{array}$ & $\begin{array}{l}\text { Amount } \\
\text { (acres) }\end{array}$ & $\begin{array}{l}\text { Shadow price } \\
\text { (dollars) }\end{array}$ & & $\begin{array}{l}\text { Amount } \\
\text { (hours) }\end{array}$ & $\begin{array}{l}\text { Shadow price } \\
\text { (dollars) }\end{array}$ & $\begin{array}{l}\text { Amount } \\
\text { (hours) }\end{array}$ & $\begin{array}{l}\text { Shadow price } \\
\text { (dollars) }\end{array}$ & \\
\hline \multicolumn{11}{|l|}{ Project data: } \\
\hline All farms & 45 & NA & 139 & NA & UN & UN & NA & UN & NA & 7,859 \\
\hline Beef/sheep farms & 40 & NA & 118 & NA & UN & UN & NA & UN & NA & 13,026 \\
\hline Beef farms & 49 & NA & 147 & NA & UN & UN & NA & UN & NA & 7,385 \\
\hline \multicolumn{11}{|l|}{ Model results: } \\
\hline$(P-1)$ & 32 & NA & 139 & 6 & 1,002 & 360 & 4 & 180 & 15 & 6,938 \\
\hline$(P-2)$ & 33 & NA & 139 & 10 & 667 & 101 & NA & 180 & 15 & 6,513 \\
\hline$(P-3)$ & 32 & NA & 86 & NA & 918 & 360 & 5 & 180 & 16 & 5,276 \\
\hline$(P-4)$ & 32 & NA & 139 & 2 & 963 & 360 & 5 & 180 & 16 & 7,151 \\
\hline$(P-5)$ & 33 & NA & 139 & 6 & 596 & 101 & NA & 180 & 15 & 6,898 \\
\hline$(P-6)$ & 32 & NA & 139 & 14 & 999 & 360 & 3 & 180 & 14 & 7,232 \\
\hline$(P-7)$ & 33 & NA & 139 & 16 & 655 & 96 & NA & 180 & 14 & 6,805 \\
\hline$(P-8)$ & 32 & NA & 86 & NA & 918 & 360 & 6 & 180 & 8 & 6,678 \\
\hline$(P-9)$ & $45^{b}$ & 4 & 45 & NA & 576 & 360 & 5 & 11 & NA & 5,478 \\
\hline$(P-10)$ & 45 & 53 & 119 & NA & 1,273 & 498 & 3 & 251 & 3 & $7,859^{d}$ \\
\hline$(P-11)$ & 45 & 62 & 139 & 11 & 769 & 93 & NA & 244 & 3 & 6,341 \\
\hline$(P-12)$ & 45 & 53 & 119 & NA & 1,273 & 498 & 3 & 251 & 3 & $7,859^{d}$ \\
\hline
\end{tabular}

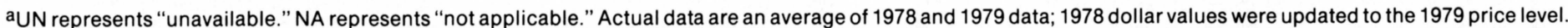

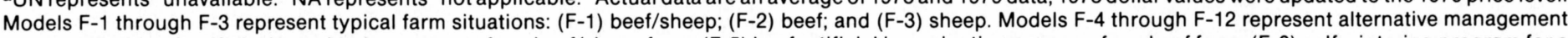

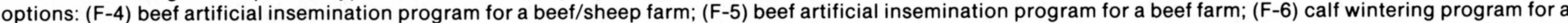

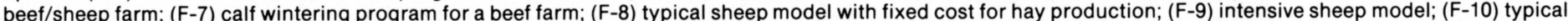

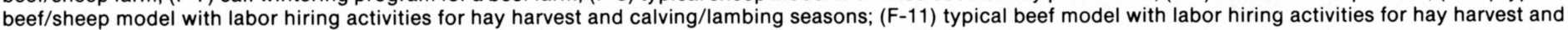
calving seasons; (F-12) typical sheep model with labor hiring activities for hay harvest and lambing seasons.

${ }^{\mathrm{b}}$ This was pasture produced on hayland.

'Since no constraint was specified in the model, no shadow price emerged. The figure represents the total amount of labor used, including hired labor.

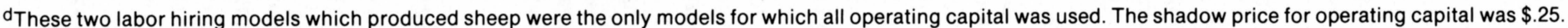


The differences between actual and modeled net income were attributed to variations in yields, resource use, enterprise combinations, and the inability of the LP models to account for the inefficiencies that exist in the real world.

In comparison to actual data, the lower level of operating capital used in the models can be attributed to several factors. Some operating costs which occur on actual farms may not have been included in the models; the average farmer may have had costs which were higher than was necessary to attain his level of output; or the LP models may have presented an optimally managed operation that, in reality, was impossible to attain in a world of imperfections.

In spite of the differences between model results and results based on average data, the authors felt that the models were sufficiently reliable to use for evaluating the economic impacts of selected alternative management strategies. However, when interpreting results, two aspects of research associated with analysis based on models of typical farms should be remembered. First, since the typical models were not perfect representations of actual situations and since the alternative models were developed from the typical models, magnitudes of changes due to the alternative management strategies should be based on comparisons with typical model results rather than comparisons with actual data. Second, it is not likely that either average actual data or typical model results will accurately represent an individual farm situation.

\section{Alternative Management Strategies}

Hypothetical budgets and LP models were prepared to estimate farm returns to fixed resources, enterprise combinations, and inputs used for four alternative management plans. It was assumed that the level of resources existed to allow for the incorporation of any one of the four alternatives.

New budgets were designed by adjusting the typical pasture and beef budgets. Nine new models, F-4 through F-12 for full-time farms and P-4 through $\mathrm{P}-12$ for part-time farms, were estimated and compared to typical farm models, F-1 through F-3 and P-1 through P-3 (Tables 4 and 5).

\section{Beef Artificial Insemination}

Assuming a hypothetical beef artificial insemination (AI) program, AI budgets were designed. Based on several studies, (Connor, Peters et al., and Inskeep and Lewis), monthly calving percentage and pregnancy rates were estimated. Due to the genetic heritibility of the bull used in an AI program, the average weight of an AI calf was 15 pounds heavier when marketed than a calf produced through natural breeding. The weights of all calves born before or after the actual calving date, March 15, were adjusted by the actual daily gain, 1.95 pounds (Baker et al.). An increased value of $\$ 7.50$ for an AI calf used as a replacement heifer was budgeted. Additional expenses associated with the AI program were $\$ 656$ for the 60 -cow full-time farm and $\$ 328$ for the 30 -cow 
part-time farm. Due to a more concentrated calving season, with AI rather than with natural breeding, the annual labor requirement per cow was reduced from 8 hours to 6 and 6.3 hours in full- and part-time AI budgets, respectively. In comparison to the full-time, 60-cow (part-time, 30-cow) beef budget with natural breeding, the AI budget reflected a decrease in total marketed calf weight of 45 (22) pounds. This decrease occurred because a portion of the cows were bred later under an AI program than with natural breeding. Cows bred later produced lighter calves by the October marketing date. The annual returns to fixed resources and feed per cow were $\$ 241.28$ and $\$ 201.48$ for fulland part-time farms, respectively.

LP models F-4 (P-4) and F-5 (P-5), reflecting changes in production activities and resource coefficients, were run and compared to typical models for the full-time (part-time) beef/sheep and beef farms, models F-1 (P-1) and F-2 (P-2), respectively. Models F-4 and F-5 incomes were within 98 and 97 percent of the typical full-time farms; models P-4 and P-5 were within 96 and 91 percent, respectively, of the typical part-time farms. Thus AI does not appear to to be more profitable than current practices, and AI is more attractive to full-time than to part-time farms. Yet, the AI income may have been understated. Compared to the $\$ 7.50$ added value per AI calf used as a replacement, Singleton and Petritz (1976) have estimated an added value as high as $\$ 15$ and Herrick's estimate was as much as $\$ 26.40$ (1976).

To maintain records on heat detection and conception rates, a producer must observe cows more closely during calving. Although this higher level of management is difficult to appraise, it is a critical area affecting profits.

\section{Calf Wintering}

The calf wintering option was designed to represent a hypothetical management system where the producer would winter one half of his raised feeder calves. Two budgets, stocker and yearling, were prepared for each farm category. Each wintered calf was fed 3 pounds of ground ear corn, 1 pound of soybean meal, and 11 pounds of non-legume hay per day to attain an average weight of 619 or 608 pounds for a full-time or a part-time farm, respectively, by a May 1 marketing date. Health care, marketing, and labor cost increases were budgeted. The full- and part-time budgets with half of the calves sold as stockers showed receipts minus cash costs of $\$ 253$ and $\$ 219$ per cow, respectively. ${ }^{12}$

The yearling budgets reflected the increase in pasture requirements and returns per cow if half of the calves would have been wintered and marketed around October 15. Starting with the initial stocker weight of 619 or 608

\footnotetext{
${ }^{12}$ Prices for stockers and yearlings relative to AHP calf prices were estimated from Kansas City market data (USDA, 1974-1984) averaged over an 11-year time period. A detailed explanation of the price determination may be found in Reda et. al. Receipts minus cash costs figures do not include costs of on-farm hay and pasture production.
} 
pounds for full- or part-time farms, respectively, and an average daily weight gain of 1.5 pounds, the receipts minus cash costs per cow equaled $\$ 311$ and $\$ 274$.

The calf wintering LP model was designed to select either a stocker program, wintering half of the calves until spring; or a yearling program, keeping wintered calves through the summer; or a combination of both half stocker and half yearling options. Models F-6, P-6, F-7, and P-7 ranged from 8 to 19 percent more profitable than the beef/sheep and beef models for both farm categories. The stocker activity was not included in the model's results; therefore, based on the data used, the option to winter calves was economically desirable only if calves were pastured after being wintered.

The profit level of this model may have been overstated because the associated enterprise budgets reflect the second year after a calf wintering program started; and the analysis did not consider the income that was postponed by initially keeping one half of the calves.

\section{Intensive Sheep}

The intensive sheep program was designed to represent a hypothetical situation in which hay production was eliminated in 1979 . Since the hay requirement was significantly greater for beef than sheep, it was thought that a farm producing sheep and buying hay might be more profitable than a combination beef/sheep operation. This deliberately planned diminution in beef production that implied product conversion towards a larger sheep flock was possibleespecially if the surplus hay land had been used for pasture, as assumed in this analysis. The objective of this study was to determine the economic feasibility of producing sheep at a level which did not exist in the Project area. The data used to budget the returns per ewe were the same as those used for the typical sheep model.

Assuming that hay land would have been more productive than pasture land, an alternative pasture budget was prepared to represent higher yields. Based on data from the AHP area (Baker, p. 173) the average annual yield per acre for all AHP farms was 6,973 pounds of dry matter. The intensive sheep models F-9 and P-9 were constructed by including a hay purchasing activity ${ }^{13}$ and a second pasture producing activity that replaced the hay producing activity.

A typical sheep plan, models F-8 and P-8, which included the fixed costs of hay production, was used as a basis of comparison to models F-9 and P-9. The full-time intensive sheep model F-9 income was 29 percent less than model F-8 while the same quantity of sheep, 186, was raised. The value of hay purchased in model F-9 was $\$ 3,115$, whereas there was no hay purchased in model F-8; thus, part of the difference in net income could be attributed to hay sales of $\$ 4,954$ in model F-8. The comparative models for the part-time group showed 39 percent less profit when purchasing hay to raise 111 sheep. Model P-8 was more profitable than model P-9 partly because of hay sales equal to $\$ 2,780$.

${ }^{13}$ The cost of purchasing a high quality mixed hay was assumed to be $\$ 56.00$ per ton. 
These results raise the question, "Should the valid model of comparison for the intensive plan include a hay selling activity?" When models F-8, and P-8 were run prohibiting hay sales, the income was respectively $\$ 5,943$ for full-time farms and $\$ 2,562$ for part-time farms. Although both of these income figures were closer to the results of the intensive sheep models than were results of model F-8 and P-8, they were still 11 and 25 percent greater, respectively. It was concluded that even if a farm is not able to sell hay, it is more profitable for a sheep farmer to produce his own hay than to buy hay.

\section{Labor Hiring}

The typical models included shadow prices ranging from $\$ 4$ to $\$ 17$ for seasonal labor. ${ }^{14}$ To test the profitability of hiring labor, it was not necessary to create any new budgets.

The final three models, F-10 through F-12 and P-10 through P-12, for a beef/sheep, beef, and sheep farm, respectively, included labor hiring activities at the 1979 hourly wage rate of $\$ 2.58$ (USDA 1981, p. 432) for the June hay harvest and calving/lambing seasons. The optimal solutions for the beef/sheep/labor model F-10 and P-10 and the sheep/labor model F-12 and P-12 were identical for both farm categories. The cow/calf activity was not selected due to the higher profitability of sheep production compared to beef production. The model for the ful1-time (part-time) category, included 882 [138] hours of hired labor for the lambing season and 86 [71] hours of hired labor for the June hay harvest season. The optimal incomes of the full-time, (part-time) beef/sheep/labor model and sheep/labor model were 37 [20] percent and $60[28]$ percent greater than the respective typical beef/sheep and typical sheep models.

The beef/labor model F-11 and P-11 results featured a net income that was approximately 8 and 20 percent greater than the typical beef model results for the full- and part-time farms, respectively. For both categories, fewer cows were included in the optimal solution than were shown in typical models. While it was profitable to hire labor during the haying season, it was not profitable to hire labor during the calving season. Thus, for the full-time model, the 8 percent increase in income can be attributed to a smaller amount of purchased hay; 62 hours of hired labor corresponding to a 23 percent increase in acres of hay production; and a 53 percent decrease in hay purchases. The part-time model's increased income was primarily the result of increased hay production; an additional 64 hours of hired labor corresponding to a 36 percent increase in acres of hay production; and a substantial increase in hay sales.

${ }^{14}$ The shadow price indicates how much returns to fixed resources could be increased by the addition of one more unit of a limited resource. 


\section{Survey of AHP Farmers}

To evaluate the potential for adoption, a telephone survey was designed to asess the 1978-79 AHP participants' reactions to the alternative management systems. To distinguish responses from those already adopting an alternative strategy, part of the questionnaire was designed to ascertain 1982 management practices.

Since there were only 56 participants in $1978-79$, an attempt was made to interview the entire population. Interviews were completed with 48 farmers, or 86 percent of the population. The remaining 14 percent could either not be contacted by telephone or were no longer farming.

\section{Marketing Beef Cattle}

Eighty-one percent of the farmers marketed 50 percent or more of their calf crop as feeder calves in the fall (Figure 1). Of the 48 farmers surveyed, 19, 1, 4, and 2 marketed 100 percent of their calf crop as calves, stockers, yearlings, or other, respectively (Table 6). The remaining 22, or 46 percent, sold their calves as a combination of two or more of the above categories. Eleven of the 22 farmers marketed their calf crop as a combination of only calves and stockers. Ten farmers sold all or part of their calf crop as yearlings. Of these 10 farmers, 8 sold 50 percent or more of their calf crop as yearlings. The most important reason cited for not keeping raised calves as yearlings was a shortage of resources, i.e., cash, feed, labor, land, buildings, or facilities. ${ }^{15}$

Ten farmers wintered 50 percent or more of their calves for sale as yearlings or older cattle. Of the remaining 38 who did not keep calves as long, 23 replied positively to the question, "Would you be willing to keep 50 percent of your raised calf crop until the following fall if it were 10-20 percent more profitable?" Of the ten who responded, "Maybe or not sure" to the same question, five said that risk or a lack of resources were the main reasons for hesitating to consider the yearling option. Five who responded "No," stated they were unwilling to consider this program because of a lack of resources only.

The cooperators' acceptance of the calf wintering option was very favorable. Some were eager to obtain more information in order to adjust their marketing strategies.

\section{Hiring Labor}

Sixty-seven percent of the respondents hired labor in 1982. The 48 farmers interviewed averaged 406 hours of hired labor per year at an average wage rate of $\$ 3.23$ per hour. The farms which hired labor during the haying season hired almost 130 hours of labor per month in June through August (Figure 2). On a

${ }^{15}$ Farmers were asked if they bought calves for resale. Twenty percent said yes. 


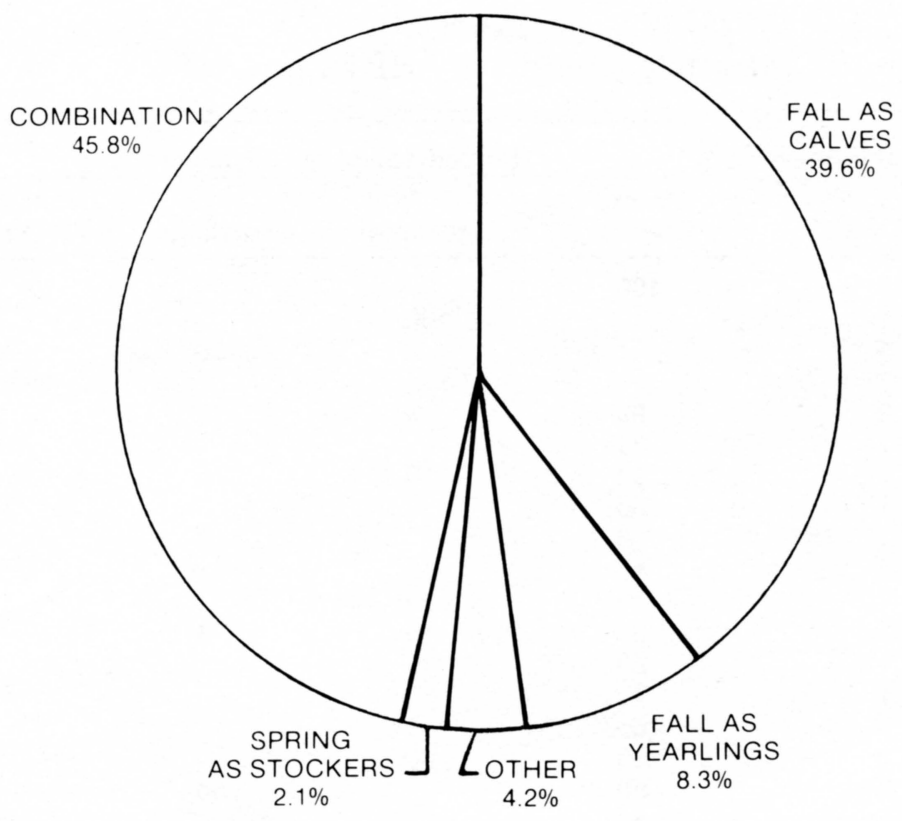

Figure 1. Marketing strategies of AHP farmers, 1982.

monthly basis, the highest incidence of hired labor occurred in June through August, presumably to harvest hay (Figure 3).

Forty percent of the respondents did not hire additional labor because they felt it cost too much. Thirty-five percent replied that they did not need additional labor. When asked if they were to hire additional labor, 56 percent of the group responded they would have labor work on normal farm chores or construction projects such as building fences and clearing brush.

Only 14 of the 48 farmers surveyed said they would be willing to hire labor to increase sheep production if their profits would increase by a third. Thirteen replied, "Maybe or not sure." Twelve of the 21 remaining farmers said they would not be willing to adopt the strategy due to personal preferences.

The cooperators' acceptance of the hiring labor option was not as favorable as that of the calf-wintering option for two reasons: (1) the cost or an insufficient need, and (2) a disinterest in hiring labor specifically to raise sheep. There was a biased or negative attitude toward raising sheep. Some made note of the predator problem associated with raising sheep while others stated they did not like sheep. Several doubted that hiring labor would increase their profits by 33 percent. The majority said they would have hired labor work on normal farm chores; however, hiring labor for this purpose was not considered to be a profit-earning activity in this study. 
Table 6.

Marketing strategies of AHP farmers, 1982.

\begin{tabular}{|c|c|c|c|c|c|}
\hline \multicolumn{2}{|c|}{ Number of farmers } & \multicolumn{4}{|c|}{ Percentage of raised calf crop } \\
\hline & & $\begin{array}{l}\text { Feeder } \\
\text { calves }\end{array}$ & Stockers & Yearlings & Other \\
\hline & 19 & 100 & & & \\
\hline & 1 & & 100 & & \\
\hline & 4 & & & 100 & \\
\hline & 2 & & & & 100 \\
\hline & 2 & 95 & 5 & & \\
\hline & 1 & 95 & & & 5 \\
\hline & 2 & 90 & 10 & & \\
\hline & 1 & 90 & & & 10 \\
\hline & 1 & 90 & & 5 & 5 \\
\hline & 1 & 85 & 15 & & \\
\hline & 1 & 80 & 20 & & \\
\hline & 1 & 80 & 15 & 5 & \\
\hline & 1 & 80 & 15 & & 5 \\
\hline & 1 & 70 & 30 & & \\
\hline & 2 & 65 & 35 & & \\
\hline & 2 & 50 & 50 & & \\
\hline & 2 & 50 & & 50 & \\
\hline & 2 & 50 & & & 50 \\
\hline & 1 & 20 & & 80 & \\
\hline & 1 & & 50 & 50 & \\
\hline Total & 48 & & & & \\
\hline
\end{tabular}

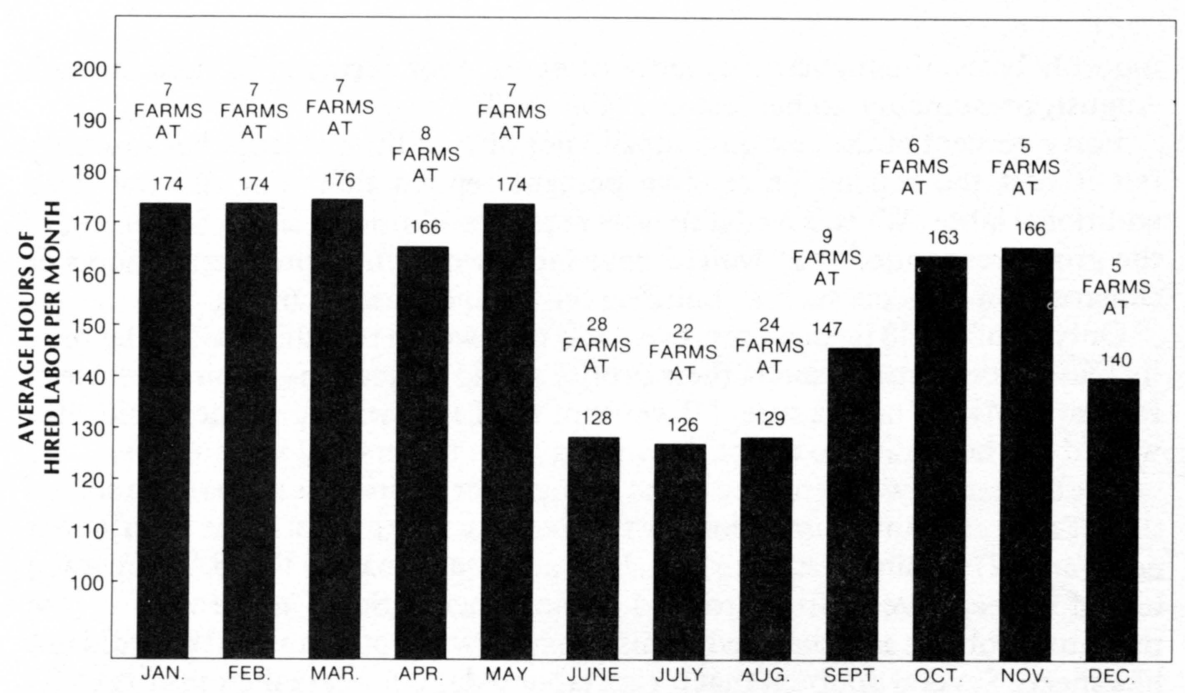

Figure 2. Number of AHP farms hiring labor and average hours of labor hired per month, 1982. 


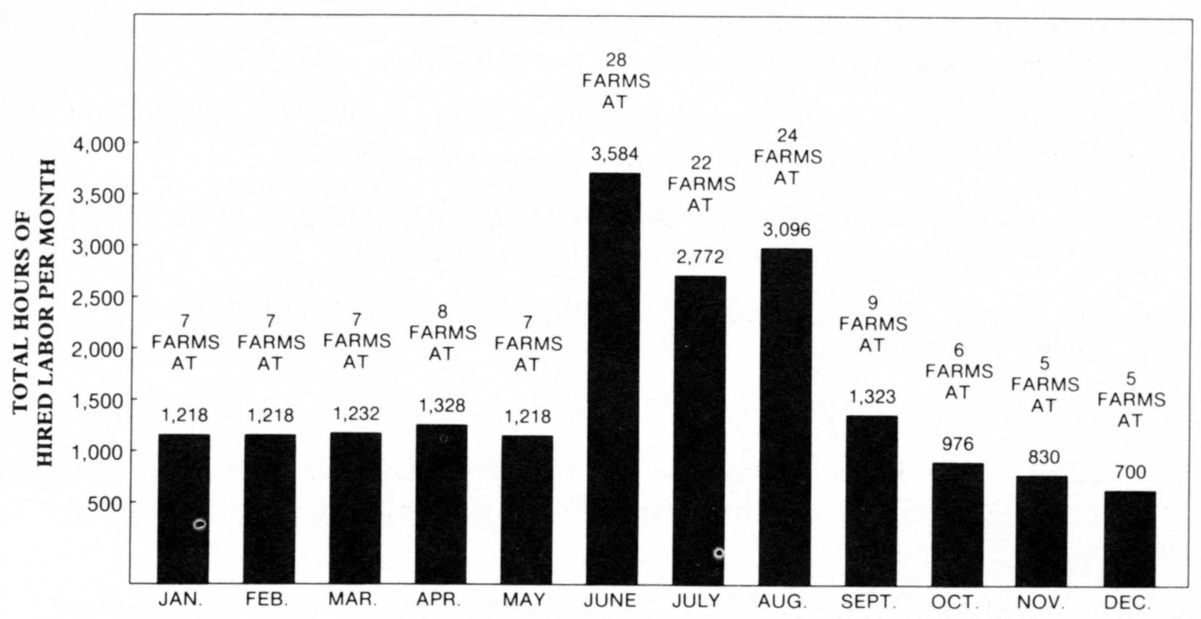

Figure 3. Number of AHP farms hiring labor and total hours of labor hired per month, 1982.

\section{Conclusions}

Two of the four alternative management strategies examined in this investigation increased returns to fixed resources. Wintering 50 percent of the calf crop for sale as yearlings and hiring seasonal labor to raise sheep were found to be significantly more profitable than activities currently being practiced on the majority of the modeled farms. Many farmers were interested in the possibility of wintering calves, but fewer farmers were interested in hiring labor to produce sheep. Beef artifical insemination and intensive sheep production with purchased hay were found to be less profitable than typical farm operations.

Results from this study indicate that opportunities exist to improve farm income through alternative management strategies. However, increasing farm income frequently depends on more intensive use of labor and capital, the addition and/or alternation of enterprises, and improved management.

\section{References}

Baker, Barton S., Paul E. Lewis, Dale K. Colyer, Frank E. Woodson, E. Keith Inskeep, and Robert H. Maxwell. Allegheny Highlands Project 1970-1979. West Virginia University Agr. and For. Exp. Sta., Dec. 1981.

Baker, Barton S. "Production and Species Composition of Hill Pasture Influenced by Lime and Fertilizer," Hill Lands: Proceedings of an International Symposium. Eds. Luchok, Cawthon and Breslin. Morgantown: West Virginia University Books, Office of Publications, 1976, pp. 171-174. 
Beneke, Raymond R., and Ronald Winterboer. Linear Programming Applications to Agriculture. Ames: Iowa State University Press, 1973.

Connor, Ann Louise. "Factors Affecting the Reproductive Performance of Beef Cattle.” M.S. thesis, West Virginia University, 1981.

Cooperative Extension Service. Farm Planning and Financial Management ID-68. West Lafayette, Indiana: Purdue University, 1980.

Crecink, John C. Characteristics of Small Farm Families. USDA EDD ESCS, Nov. 1979.

Herrick, J. B. "Your Best Buy Is A. I.” Curtiss BeefCattletter. No. 8(1976), p. 1.

Inskeep, E. K., and Paul Lewis. Use of Prostaglandin $F_{2}$ and EB in Beef Cattle Management. West Virginia University, Coll. Agr., Div. An. and Vet. Sci. (not dated).

Orden, David and Dennis K. Smith. Small Farm Programs: Implications Froma Study in Virginia. Research Division Bull. 135. Blacksburg, Virginia: Virginia Polytechnic Institute and State University, Oct. 1978.

Peters, John B., J. A. Welch, A. L. Barr, and E. K. Inskeep. "Synchronization Estrus and Use of Artificial Insemination in Small Herds of Beef Cattle," Hill Lands: Proceedings of an International Symposium. Eds. Luchok, Cawthon, and Breslin. Morgantown: West Virginia University Books, Office of Publications, 1976, pp. 631-634.

Reda, Kimberly Jane. "An Economic Analysis of Management Strategies on Small, Beef/Sheep Farms in West Virginia." M.S. thesis, West Virginia University, 1984.

Reda-Wilson, Kimberly, Robert O. Burton, Jr., Barton S. Baker, and Paul E. Lewis. Wintering Calves: An Analysis of Potential Returns on West Virginia Farms. Circular 136. West Virginia University Agr. and For. Exp. Sta., Oct. 1985.

Singleton, W. L., and D. C. Petritz. "Management and Economics of a Beef Cow Herd AI Program.” The Charolois Way, May 1976, pp. 55-65.

U.S. Department of Agriculture. Agricultural Statistics 1981. Washington: U.S. Government Printing Office, 1981.

U.S. Department of Agriculture. Livestock Detailed Quotations. AMS LPGS214, Various Issues 1974-1984.

U.S. Department of Agriculture Cooperating with the West Virginia Department of Agriculture. West Virginia Agricultural Statistics, 1982. CRB 13, Nov. 1982.

U.S. Department of Commerce, Bureau of the Census. 1978 Census of Agriculture, Volume I, State and County Data, Part 48, West Virginia. Washington, 1981. 


\section{Appendix}

Table A-1

AHP variable descriptions and mean values.

\begin{tabular}{llrr}
\hline \hline Description (data classification)a & Unit & $\begin{array}{r}\text { Full-time } \\
\text { farms }\end{array}$ & $\begin{array}{r}\text { Part-time } \\
\text { farms }\end{array}$ \\
\hline Acres of pasture per animal unit & Acres & 3.20 & 3.60 \\
Number of cows and bred heifers & Head & 66.34 & 32.46 \\
Electricity expense & Dollars & 222.81 & 174.45 \\
Number of ewes and bred lambs & Head & 136.79 & 55.78 \\
Income from sale of hay & Dollars & 217.86 & 218.63 \\
Hay produced per acre (dry matter) & Tons & 2.88 & 2.76 \\
Calves marketed per cow & Percent & 87.68 & 85.67 \\
Lambs marketed per ewe & Percent & 120.10 & 121.31 \\
Market weight per calf & Pounds & 483.25 & 472.33 \\
Total operating receipts & Dollars & 32871.37 & 9678.35 \\
Number of pasture acres & Acres & 209.60 & 94.05 \\
Percentage of cows that died during the & & & \\
$\quad$ year & Percent & 1.73 & 1.86 \\
Percentage of ewes that died during the & & & \\
$\quad$ year & Percent & 7.83 & 10.97 \\
Expenses for purchased hay & Dollars & 636.37 & 172.14 \\
Expenses for repairs to real estate & Dollars & 869.82 & 439.72 \\
Expenses for salt \& other minerals & Dollars & 304.70 & 104.21 \\
Telephone expense & Dollars & 190.40 & 140.90 \\
Number of tillable acres & Acres & 65.48 & 45.38 \\
Total operating expenses & Dollars & 24764.54 & 7859.33 \\
Total animal units & Animal & & \\
& unit & 109.15 & 43.90 \\
Veterinary and drug expenses & Dollars & 654.69 & 176.89 \\
Dollar value per lamb sold & Dollars & 58.75 & 52.64 \\
Dollar value per calf sold & Dollars & 348.88 & 334.43 \\
Market weight per lamb & Pounds & 96.95 & 89.56 \\
\hline & & & \\
\hline
\end{tabular}

aData were coded and stored at the Division of Resource Management, West Virginia University. Missing data were ignored when calculating means. 
Table A-2

Machinery inventory for twenty-six full-time AHP farms.

\begin{tabular}{|c|c|c|c|c|}
\hline $\begin{array}{l}\text { Machinery } \\
\text { category }\end{array}$ & $\begin{array}{l}\text { Total for } \\
\text { all farms }\end{array}$ & $\begin{array}{l}\text { Average } \\
\text { number } \\
\text { per farm }\end{array}$ & $\begin{array}{l}\text { Pieces of } \\
\text { machinery per } \\
\text { farm, by numbers } \\
\text { of farms }\end{array}$ & $\begin{array}{l}\text { Type of machine } \\
\text { (pieces of machin- } \\
\text { ery per farm by } \\
\text { number of farms) }\end{array}$ \\
\hline Balers & 32 & 1.20 & $\begin{array}{r}8 \text { farms }-2 \\
16 \text { farms }-1 \\
2 \text { farms }-0\end{array}$ & UN \\
\hline $\begin{array}{l}\text { Corn } \\
\text { harvesting } \\
\text { equipment }\end{array}$ & 25 & .96 & $\begin{array}{l}7 \text { farms }-2 \text { or } 3 \\
7 \text { farms }-1 \\
12 \text { farms }-0\end{array}$ & $\begin{array}{l}10 \text { choppers } \\
9 \text { corn pickers } \\
6 \text { ensilage blowers }\end{array}$ \\
\hline $\begin{array}{l}\text { Small } \\
\text { equipment }\end{array}$ & 75 & 2.90 & $\begin{array}{l}1 \text { farm }-13 \\
13 \text { farms }-2 \text { to } 12 \\
12 \text { farms }-0 \text { or } 1\end{array}$ & $\begin{array}{l}30 \text { saws } \\
10 \text { sprayers }\end{array}$ \\
\hline $\begin{array}{l}\text { Large } \\
\text { equipment } \\
\text { used } \\
\text { with other } \\
\text { machinery }\end{array}$ & 157 & 6.04 & $\begin{array}{c}1 \text { farm }-17 \\
17 \text { farms }-4 \text { to } 16 \\
8 \text { farms }-0 \text { to } 3\end{array}$ & $\begin{array}{l}49 \text { spreaders } \\
16 \text { post drivers } \\
13 \text { brush hogs } \\
9 \text { blades }\end{array}$ \\
\hline $\begin{array}{l}\text { Farm and } \\
\text { recreational } \\
\text { vehicles }\end{array}$ & 10 & .38 & $\begin{array}{l}6 \text { farms }-1 \text { or more } \\
20 \text { farms }-0\end{array}$ & $\begin{array}{l}7 \text { motor bikes } \\
3 \text { snowmobiles }\end{array}$ \\
\hline \multirow[t]{3}{*}{$\begin{array}{l}\text { Hay } \\
\text { harvesting }\end{array}$} & 105 & 4.04 & $\begin{array}{c}1 \text { farm }-10 \\
11 \text { farms }-4 \text { to } 9 \\
13 \text { farms }-1 \text { to } 3 \\
1 \text { farm }-0\end{array}$ & $\begin{array}{l}34 \text { mowers } \\
(1 \text { farm }-8) \\
(6 \text { farms }-2) \\
(14 \text { farms }-1) \\
(5 \text { farms }-0)\end{array}$ \\
\hline & & & & $\begin{array}{l}21 \text { rakes } \\
(2 \text { farms }-2) \\
(17 \text { farms }-1) \\
(7 \text { farms }-0)\end{array}$ \\
\hline & & & & $\begin{array}{l}19 \text { tedders } \\
(1 \text { farm-3) } \\
(1 \text { farm-2) } \\
(14 \text { farms }-1) \\
(10 \text { farms }-0)\end{array}$ \\
\hline
\end{tabular}

Continued 


\begin{tabular}{|c|c|c|c|c|}
\hline $\begin{array}{l}\text { Machinery } \\
\text { category }\end{array}$ & $\begin{array}{l}\text { Total for } \\
\text { all farms }\end{array}$ & $\begin{array}{l}\text { Average } \\
\text { number } \\
\text { per farm }\end{array}$ & $\begin{array}{l}\text { Pieces of } \\
\text { machinery per } \\
\text { farm, by numbers } \\
\text { of farms }\end{array}$ & $\begin{array}{l}\text { Type of machine } \\
\text { (pieces of machin- } \\
\text { ery per farm by } \\
\text { number of farms) }\end{array}$ \\
\hline & & & & $\begin{array}{l}14 \text { haybines } \\
(1 \text { farm-2) } \\
(12 \text { farms }-1) \\
(13 \text { farms }-0)\end{array}$ \\
\hline & & & & $\begin{array}{l}11 \text { conditioners } \\
(1 \text { farm-2) } \\
(9 \text { farms }-1) \\
(16 \text { farms }-0)\end{array}$ \\
\hline $\begin{array}{l}\text { Miscellaneous } \\
\text { equipment }\end{array}$ & 130 & 5 & $\begin{array}{l}3 \text { farms }-15,20,23 \\
9 \text { farms }-5 \text { to } 14 \\
14 \text { farms }-1 \text { to } 4\end{array}$ & $\begin{array}{l}42 \text { watering or } \\
\text { feeding } \\
\text { equipment } \\
17 \text { elevators }\end{array}$ \\
\hline $\begin{array}{l}\text { Road } \\
\text { vehicles }\end{array}$ & 71.5 & 2.75 & $\begin{array}{c}1 \text { farm }-7 \\
16 \text { farms }-3 \text { to } 6 \\
8 \text { farms }-1 \text { or } 2 \\
1 \text { farm }-0\end{array}$ & $\begin{array}{l}\text { UN } \\
\text { UN } \\
\text { UN } \\
\text { UN }\end{array}$ \\
\hline $\begin{array}{l}\text { Seeding } \\
\text { equipment }\end{array}$ & 23 & .88 & $\begin{array}{r}7 \text { farms }-2 \\
9 \text { farms }-1 \\
10 \text { farms }-0\end{array}$ & $\begin{array}{l}9 \text { corn } \\
\text { planters } \\
9 \text { drills }\end{array}$ \\
\hline $\begin{array}{l}\text { Tillage } \\
\text { implements }\end{array}$ & 65 & 2.5 & $\begin{array}{l}1 \text { farm }-7 \\
7 \text { farms }-4 \text { to } 6 \\
12 \text { farms }-1 \text { to } 3 \\
6 \text { farms }-0\end{array}$ & $\begin{array}{l}26 \text { disks } \\
(2 \text { farms-3) } \\
\text { ( } 2 \text { farms }-2) \\
(13 \text { farms }-1) \\
(9 \text { farms }-0)\end{array}$ \\
\hline & & & & $\begin{array}{l}23 \text { plows } \\
\text { ( } 1 \text { farm-3) } \\
\text { ( } 5 \text { farms-2) } \\
(13 \text { farms }-1) \\
(7 \text { farms }-0)\end{array}$ \\
\hline Tractors & 73 & 2.8 & $\begin{array}{c}1 \text { farm }-6 \\
23 \text { farms }-1 \text { to } 5 \\
2 \text { farms }-0\end{array}$ & $\begin{array}{l}\text { UN } \\
\text { UN } \\
\text { UN }\end{array}$ \\
\hline
\end{tabular}


Table A-2 continued

\begin{tabular}{|c|c|c|c|c|}
\hline $\begin{array}{l}\text { Machinery } \\
\text { category }\end{array}$ & $\begin{array}{l}\text { Total for } \\
\text { all farms }\end{array}$ & $\begin{array}{l}\text { Average } \\
\text { number } \\
\text { per farm }\end{array}$ & $\begin{array}{l}\text { Pieces of } \\
\text { machinery per } \\
\text { farm, by numbers } \\
\text { of farms }\end{array}$ & $\begin{array}{l}\text { Type of machine } \\
\text { (pieces of machin- } \\
\text { ery per farm by } \\
\text { number of farms) }\end{array}$ \\
\hline Wagon & 48 & 1.8 & $\begin{array}{c}2 \text { farms }-6 \\
6 \text { farms }-4 \text { or } 5 \\
18 \text { farms }-1 \text { to } 3\end{array}$ & $\begin{array}{l}\text { UN } \\
\text { UN } \\
\text { UN }\end{array}$ \\
\hline Total & 824 & 32 & $\begin{array}{l}1 \text { farm }-70 \\
1 \text { farm }-4\end{array}$ & $\begin{array}{l}\text { UN } \\
\text { UN }\end{array}$ \\
\hline
\end{tabular}

UN represents "unavailable."

\section{Table A-3}

Machinery inventory for thirty part-time AHP farms.

\begin{tabular}{|c|c|c|c|c|}
\hline $\begin{array}{l}\text { Machinery } \\
\text { category }\end{array}$ & $\begin{array}{l}\text { Total for } \\
\text { all farms }\end{array}$ & $\begin{array}{l}\text { Average } \\
\text { number } \\
\text { per farm }\end{array}$ & $\begin{array}{l}\text { Pieces of } \\
\text { machinery per } \\
\text { farm, by numbers } \\
\text { of farms }\end{array}$ & $\begin{array}{l}\text { Type of machine } \\
\text { (pieces of machin- } \\
\text { ery per farm by } \\
\text { number of farms) }\end{array}$ \\
\hline Balers & 31 & 1.02 & $\begin{array}{l}6 \text { farms }-2 \text { or more } \\
18 \text { farms }-1 \\
6 \text { farms }-0\end{array}$ & UN \\
\hline $\begin{array}{l}\text { Corn } \\
\text { harvesting } \\
\text { equipment }\end{array}$ & 16 & .53 & $\begin{array}{l}8 \text { farms }-1 \text { or more } \\
22 \text { farms }-0\end{array}$ & $\begin{array}{l}8 \text { choppers } \\
4 \text { pickers }\end{array}$ \\
\hline $\begin{array}{l}\text { Small } \\
\text { equipment }\end{array}$ & 62 & 2.07 & $\begin{array}{l}2 \text { farms }-8 \\
11 \text { farms }-2 \text { to } 7 \\
17 \text { farms }-0 \text { or } 1\end{array}$ & $\begin{array}{l}20 \text { saws } \\
6 \text { sprayers }\end{array}$ \\
\hline $\begin{array}{l}\text { Large } \\
\text { equipment } \\
\text { used } \\
\text { with other } \\
\text { machinery }\end{array}$ & 118 & 3.93 & $\begin{array}{l}1 \text { farm }-10 \\
13 \text { farms }-4 \text { to } 9 \\
16 \text { farms }-0 \text { to } 3\end{array}$ & $\begin{array}{l}40 \text { spreaders } \\
15 \text { blades } \\
11 \text { brush hogs } \\
6 \text { post drivers } \\
6 \text { post diggers }\end{array}$ \\
\hline
\end{tabular}

Continued 


\begin{tabular}{|c|c|c|c|c|}
\hline $\begin{array}{l}\text { Machinery } \\
\text { category }\end{array}$ & $\begin{array}{l}\text { Total for } \\
\text { all farms }\end{array}$ & $\begin{array}{l}\text { Average } \\
\text { number } \\
\text { per farm }\end{array}$ & $\begin{array}{l}\text { Pieces of } \\
\text { machinery per } \\
\text { farm, by numbers } \\
\text { of farms }\end{array}$ & $\begin{array}{l}\text { Type of Machine } \\
\text { (pieces of machin- } \\
\text { ery per farm by } \\
\text { number of farms) }\end{array}$ \\
\hline $\begin{array}{l}\text { Farm and } \\
\text { recreational } \\
\text { vehicles }\end{array}$ & 13 & .43 & $\begin{array}{l}5 \text { farms }-1 \text { or more } \\
25 \text { farms }-0\end{array}$ & $\begin{array}{l}12 \text { motor bikes } \\
1 \text { camper }\end{array}$ \\
\hline \multirow[t]{4}{*}{$\begin{array}{l}\text { Hay } \\
\text { harvesting } \\
\text { equipment }\end{array}$} & 89 & 2.96 & $\begin{array}{c}1 \text { farm }-8 \\
7 \text { farms }-4 \text { to } 7 \\
19 \text { farms }-1 \text { to } 3 \\
3 \text { farms }-0\end{array}$ & $\begin{array}{l}33 \text { mowers } \\
(1 \text { farm-3) } \\
(8 \text { farms }-2) \\
(14 \text { farms }-1) \\
(10 \text { farms }-0)\end{array}$ \\
\hline & & & & $\begin{array}{l}25 \text { rakes } \\
(1 \text { farm-4) } \\
(2 \text { farms }-2) \\
(17 \text { farms }-1) \\
(10 \text { farms }-0)\end{array}$ \\
\hline & & & & $\begin{array}{l}12 \text { conditioners } \\
(2 \text { farms }-2) \\
(8 \text { farms }-1) \\
(20 \text { farms }-0)\end{array}$ \\
\hline & & & & $\begin{array}{l}11 \text { tedders } \\
(1 \text { farm-2) } \\
(9 \text { farms }-1) \\
(20 \text { farms }-0)\end{array}$ \\
\hline $\begin{array}{c}\text { Miscellaneous } \\
\text { equipment }\end{array}$ & 51 & 1.70 & $\begin{array}{l}1 \text { farm }-9 \\
1 \text { farm }-6 \\
20 \text { farms }-1 \text { to } 4 \\
8 \text { farms }-0\end{array}$ & $\begin{array}{l}12 \text { elevators } \\
12 \text { feeding } \\
\text { equipment }\end{array}$ \\
\hline $\begin{array}{l}\text { Road } \\
\text { vehicles }\end{array}$ & 44 & 1.47 & $\begin{array}{l}1 \text { farm }-5 \\
28 \text { farms }-1 \text { or } 3 \\
1 \text { farm }-0\end{array}$ & $\begin{array}{l}\text { UN } \\
\text { UN } \\
\text { UN }\end{array}$ \\
\hline $\begin{array}{l}\text { Seeding } \\
\text { equipment }\end{array}$ & 16 & .53 & $\begin{array}{c}1 \text { farm }-3 \\
2 \text { farms }-2 \\
9 \text { farms }-1 \\
18 \text { farms-0 }\end{array}$ & $\begin{array}{l}8 \text { drills } \\
5 \text { corn planters }\end{array}$ \\
\hline
\end{tabular}


Table A-3 continued

\begin{tabular}{|c|c|c|c|c|}
\hline $\begin{array}{l}\text { Machinery } \\
\text { category }\end{array}$ & $\begin{array}{l}\text { Total for } \\
\text { all farms }\end{array}$ & $\begin{array}{l}\text { Average } \\
\text { number } \\
\text { per farm }\end{array}$ & $\begin{array}{l}\text { Pieces of } \\
\text { machinery per } \\
\text { farm, by numbers } \\
\text { of farms }\end{array}$ & $\begin{array}{l}\text { Type of Machine } \\
\text { (pieces of machin- } \\
\text { ery per farm by } \\
\text { number of farms) }\end{array}$ \\
\hline $\begin{array}{l}\text { Tillage } \\
\text { implements }\end{array}$ & 51 & 1.7 & $\begin{array}{l}3 \text { farms }-4 \\
21 \text { farms }-1 \text { to } 3 \\
6 \text { farms }-0\end{array}$ & 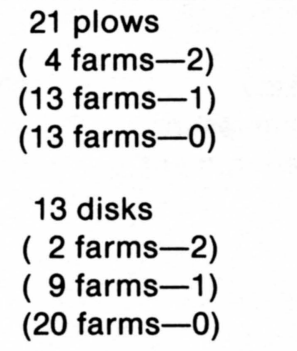 \\
\hline Tractors & 61 & 2.03 & $\begin{array}{l}1 \text { farm }-4 \\
27 \text { farms }-1 \text { to } 3 \\
2 \text { farms }-0\end{array}$ & $\begin{array}{l}\text { UN } \\
\text { UN } \\
\text { UN }\end{array}$ \\
\hline Wagons & 35 & 1.17 & $\begin{array}{l}2 \text { farms }-4 \\
17 \text { farms }-1 \text { to } 3 \\
11 \text { farms }-0\end{array}$ & UN \\
\hline Total & 585 & 19.5 & $\begin{array}{c}1 \text { farm }-36 \\
28 \text { farms }-2 \text { to } 35 \\
1 \text { farm }-1\end{array}$ & UN \\
\hline
\end{tabular}

UN represents "unavailable." 
[Blank Page in Original Bulletin] 